\title{
Dietary histidine supplementation prevents cataract development in adult Atlantic salmon, Salmo salar L., in seawater
}

\author{
R. Waagb $\varnothing^{1 *}$, C. Tröße ${ }^{1}$, W. Koppe ${ }^{2}$, R. Fontanillas ${ }^{2}$ and O. Breck ${ }^{3}$ \\ ${ }^{1}$ National Institute of Nutrition and Seafood Research (NIFES), PO Box 2029, Nordnes, N-5817 Bergen, Norway \\ ${ }^{2}$ Skretting Aquaculture Research Center, ARC, Stavanger, Norway \\ ${ }^{3}$ Marine Harvest Norway, Bergen, Norway
}

(Received 7 January 2010 - Revised 24 May 2010 - Accepted 25 May 2010 - First published online 9 August 2010)

The aim of the present study was to investigate the cataract preventive effect of dietary histidine regimes in adult Atlantic salmon (Salmo salar L.) in seawater, both through manipulating the dietary histidine level and feeding period. Mean body weight of individually tagged Atlantic salmon at the start of the experiment was 1662 (SD 333) g. Low prevalence of mild cataracts were recorded in the beginning of June. Three fishmeal and fish oil-based extruded diets (crude protein: $375 \mathrm{~g} / \mathrm{kg}$ and fat: $342 \mathrm{~g} / \mathrm{kg}$ ), differing only in histidine content (low (L): 9.3, medium (M): $12 \cdot 8$ and high (H): $17.2 \mathrm{~g}$ histidine/ $\mathrm{kg}$ diets), were fed to duplicate net pens in seawater. The experimental period was divided into three seasons (June-July; July-September; September-October), each starting and ending with individual cataract examination, assessment of somatic data, and sampling of lens and muscle tissues for analysis of histidine and histidine derivatives. In July and September, a part of the population fed L-and H-histidine feeds were transferred (crossed over) to respective series of replicate net pens fed L-, M- and H-histidine diets (i.e. eleven experimental feeding groups at trial conclusion). The fish doubled their body weight from June to October, with no systematic effects on weight gain of dietary histidine feeding regimes. Development of severe cataracts was observed between July and September. The cataract severity was directly related to the dietary histidine level fed during the first and second periods. Feeding histidine-supplemented diets ( $\mathrm{M}$ or $\mathrm{H}$ ) in the first period from June to July mitigated later cataract outbreaks. The status of selected free imidazoles in muscle and lens tissues reflected the dietary histidine feeding regimes, relative to both feed concentration and feeding duration. The study shows the risk for cataract development for adult Atlantic salmon, 1 year after the transfer of salmon smolts from freshwater to seawater, which to a major extent can be prevented by histidine supplementation just before and during the early phase of cataract development.

Atlantic salmon: Cataract: Fish welfare: Histidine: $N$-acetyl-histidine: Amino acids

The eye disorder cataract has been a relatively common observed symptom in the fish farming history, related to environmental, genetic, infectious, toxicological and nutritional factors ${ }^{(1,2)}$. Cataracts in Atlantic salmon smolts have been observed in European aquaculture for the last 20 years, and an epidemiological survey performed in 1998 in seawater farms along the Norwegian coast found a cataract prevalence as high as $82 \%^{(3)}$. The losses for the aquaculture industry vary and can be considerably high in more severe seasons ${ }^{(4)}$. Cataracts have lately also been observed in salmon growers $(>1.5 \mathrm{~kg})$ in their second year in seawater. Besides being an important ethical welfare issue for the fish farming industry, cataract development in the final phase of salmon production may also represent economical losses from reduced growth, reduced feed efficiencies, secondary infectious diseases and quality down grading at slaughter ${ }^{(4)}$.

The amino acid histidine has been identified as a key preventative factor in the development of cataract in Atlantic salmon (Salmo salar L.) smolts ${ }^{(5-7)}$. The type of cataract that occurred in the nineties at high incidences and severities in farmed salmon smolts was probably caused by the omission of blood meal from commercial salmon diets ${ }^{(8)}$. Blood meal was banned as feed ingredient as a measure to prevent the spread of bovine spongiform encephalopathy ${ }^{(9)}$. Blood meal is especially rich in histidine (approximately $50 \mathrm{~g}$ histidine/ $\mathrm{kg}$ in spray-dried products $)^{(10)}$. Breck et al. ${ }^{(6)}$ showed that feed could be supplemented with histidine either in the form of blood meal, high histidine fishmeal or as a pure histidine additive, and that all forms prevented cataract development in salmon smolts equally well.

The exact mechanism for the cataract mitigation by elevated dietary histidine level is not completely understood. It has become clear that histidine and related compounds (imidazoles) cover many important biochemical roles in the salmon tissues besides its function as essential amino acid in protein synthesis, such as components related to lens osmoregulation $^{(11,12)}$, muscle $\mathrm{pH}$ buffering ${ }^{(13,14)}$, tissue antioxidation ${ }^{(15,16)}$ and detoxification of cytotoxic-reactive carbonyl species ${ }^{(17,18)}$.

The smoltification phase in salmonids has been considered as a risk period for cataract development ${ }^{(7,8)}$. Muscle histidine

Abbreviations: H, high histidine diet; L, low histidine diet; M, medium histidine diet; NAH, $N$-acetyl histidine.

* Corresponding author: Professor R. Waagbø, fax +47 55905299, email rwa@nifes.no 
conventional Association of Official Analytical Chemists methods (1995). Feed amino acid profiles (Table 1) were analysed at Skretting ARC according to an European Union standard method ${ }^{(23)}$.

The experimental period from June to October was divided into three seasons ((June 6-July 14 (6 weeks), July 17-September 6 (13 weeks) and September 7-October 12 (18 weeks)), each starting and ending with eye examination for cataract (slit lamp biomicroscopy), assessment of somatic data (body weight, length and condition factor), sampling of lens and muscle tissues for analysis of histidine and histidine derivatives and gene expression analyses.

Approximately, 200 individuals were allocated to each net pen belonging to $\mathrm{L}$ and $\mathrm{H}$ histidine groups, while the replicates of $\mathrm{M}$ histidine groups were housed each with eighty fish. Within respective replicates, approximately thirty fish fed L-histidine feed were transferred (crossed over) to $\mathrm{M}$ (group only receiving fish) and $\mathrm{H}$ histidine dietary groups at the samplings in July and September. Similarly, thirty fish fed high-histidine feed were transferred (crossed over) to $M$ and L-histidine dietary groups at the samplings in July and September; ending up with seven dietary groups in the second period (LL, LM, LH, MM, HL, HM and HH) and eleven experimental groups in the third period (LLL, HLL, HHL, LLM, LMM, MMM, HMM, HHM, LLH, LHH and HHH) of approximately similar size, but varying in histidine level and feeding period. Fig. 1 details the experimental design with movement of fish. Since the $\mathrm{M}$ dietary groups received fish only during the two crossovers, they were housed with fewer fish at the start; meaning that all the net pens would have approximately equal biomass in the course of the trial.

The fish were exposed to a natural light regime in seawater (mean salinity of $30 \mathrm{~g} / \mathrm{l}$ ). Water temperature $(4 \mathrm{~m}$ depth) rose from 12 to $18 \cdot 5^{\circ} \mathrm{C}$ from June to July, and declined slowly to $16^{\circ} \mathrm{C}$ in the end of September and further declined to $14.4^{\circ} \mathrm{C}$

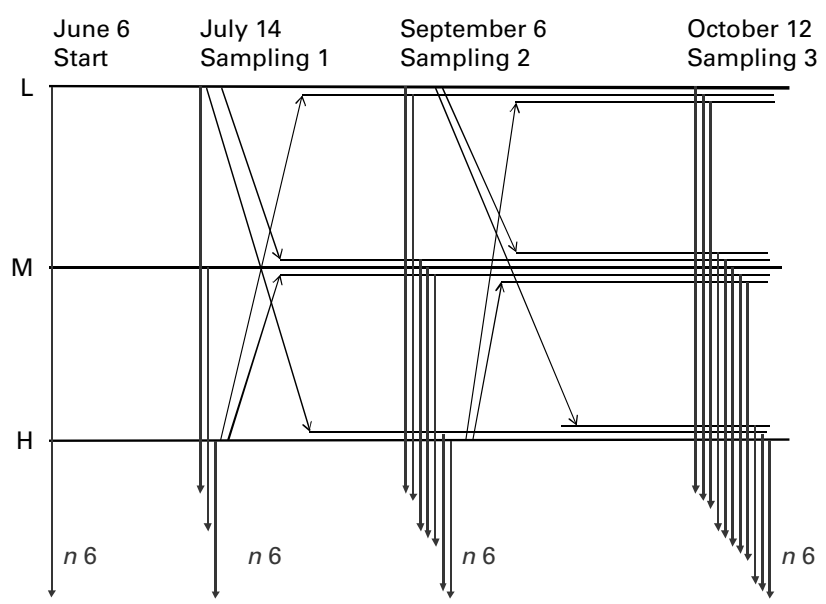

Fig. 1. Details of the experimental design run in duplicate net pens in seawater. Experimental feeds with low (L: $9.3 \mathrm{~g} / \mathrm{kg}$ ), medium (M: $12.8 \mathrm{~g} / \mathrm{kg}$ ) and high $(\mathrm{H}: 17.2 \mathrm{~g} / \mathrm{kg})$ histidine were fed during three periods, each ending with assessment of biological somatic performance data (thirty individuals per feed replicate), cataract examination (thirty per feed replicate) and tissue sampling (six per feed replicate). At samplings in July and September, individually marked fish from $\mathrm{L}$ and $\mathrm{H}$ were transferred to respective replicates of $\mathrm{L}, \mathrm{M}$ and $\mathrm{H}$ according to a crossover design, ending up with combinations of seven (LL, LM, LH, MM, HL, HM and HH) and eleven (LLL, LLM, LLH, LMM, $\mathrm{LHH}, \mathrm{MMM}, \mathrm{HLL}, \mathrm{HMM}, \mathrm{HHL}, \mathrm{HHM}$ and $\mathrm{HHH}$ ) dietary groups. at the end of October. Water oxygen concentration measured at regular intervals was never below $8.2 \mathrm{mg} / \mathrm{l}$.

The Lerang Research Station is approved, and the trial was conducted according to the guidelines of the Norwegian State Commission for Laboratory Animals.

\section{Cataract examination}

When establishing the six experimental groups, sixty fish from the two acclimatisation net pens were anaesthetised (metacain was added to the sampling tray according to an in-house protocol), weighed, measured and examined for cataract using a slit lamp biomicroscope at $15 \times$ magnification. The fish were inspected and scored individually for changes $(0-4$ per eye; i.e. $0-8$ for both the eyes per fish) according to the previously described procedure ${ }^{(24)}$. Fish with visual physical eye damages were excluded from the experiment. At later examinations (July 15, September 5 and October 12), thirty fish per replicate ( $n 60$ per dietary treatment) from all the experimental groups were examined according to the same procedure. The examined fish, except six taken for sampling, were returned to their respective net pens.

\section{Samplings}

Initially (June 6), and at July 15, September 5 and October 12, muscle and lens tissues of six fish were sampled per replicate (n 12 per dietary group) for analysis of histidine compounds and gene expression profiling ${ }^{(25)}$. The fish were fasted for $1 \mathrm{~d}$ before sampling. Individual fish were anaesthetised, inspected for cataract, measured for weight and length ( $n 30$ per replicate), and sampled for lens and muscle tissues ( $n 6$ per replicate). Skin-free epaxial white muscle tissue was sampled from behind the dorsal fin and was frozen directly on dry ice. Lenses were carefully dissected, gently rolled on clean filter paper to remove liquid and muscle attachments, immediately frozen on liquid nitrogen/dry ice, and stored at $-80^{\circ} \mathrm{C}$ until analysis.

\section{Analysis of free amino acids}

Individual muscle samples of six fish were analysed for free amino acids, while individual lenses were analysed for histidine and NAH. Free amino acids were analysed using a Biochrom 20 Plus Amino Acid Analyser (Amersham, Cambridge, UK), according to a standardised procedure from the manufacturer (Biochrom AAAFAQ08), with post-column ninhydrin derivatisation and colorimetric detection at 570 and $440 \mathrm{~nm}$. NAH and free histidine in the lens were analysed by reverse phase HPLC and UV detection at $210 \mathrm{~nm}^{(26)}$, with modifications according to Breck et al. ${ }^{(7)}$.

\section{Statistical analyses}

The data were analysed by the CSS:Statistica for Windows ${ }^{\mathrm{TM}}$ Statistical Software (version 9.0; Statsoft, Inc., Tulsa, OK, USA, 1984-9). Differences between groups were assessed by nested ANOVA (net pen individuals nested in dietary groups) and Tukey's honestly significant difference post hoc test at a level of $P<0.05$. All values are given as mean values with their standard errors if not stated otherwise. 


\section{Results}

\section{Growth and mortality}

There were no systematic differences in somatic data related to dietary histidine regimes at any samplings (Tables 2 and 3). In the first period, the fish grew from $1662 \mathrm{~g}$ (mean values; $n$ 56-58 fish from two replicates) to 2251 (SEM 62), 2135 (SEM 61) and 2200 (SEM 59) $\mathrm{g}$ in the L, M and $\mathrm{H}$ histidine groups, respectively (nested ANOVA, $P>0.05$ ). Similarly, no differences in condition factor were observed, ranging from $1 \cdot 17$ to $1 \cdot 18$; neither were any difference in growth and somatic data observed in the second period, with mean body weight ranging between 2361 and $2652 \mathrm{~g}$. At the conclusion of the trial, fish continuously fed on the high-histidine diet $(\mathrm{HHH})$ were significantly heavier than fish fed continuously on the M-histidine diet (MMM) and the crossover diet (HMM) (nested ANOVA, $P<0 \cdot 05)$. There were, however, no differences in the length (range of means from 60.7 to $63.1 \mathrm{~cm}$ ) and condition factor (range of means from 1.18 to 1.26) between the groups. Excluding the sexually mature individuals recorded at the samplings (18.5\% in September and $28 \%$ in October) improved the mean individual specific growth rate $(0.4-0.5 \% / \mathrm{d})$ in the dietary groups, but did not change the growth results relative to dietary histidine regimes.

\section{Cataract assessment}

At the start of the experiment, minor changes in one eye (score 1) was observed in sixteen fish, two fish showed score 2, and one individual was totally blind (score 8), ending with a cataract prevalence of $30 \%$ and a mean score of 0.4 of sixty-three inspected fish. A similar overall outcome was observed in July, with approximately $30 \%$ cataracts in the fish population. All the groups showed very mild cataract changes, with no difference in incidence and mean cataract scores ( $\leq 0.7$; Fig. 2).

From July to September, a major outbreak of cataracts was recorded, with mean cataract scores reflecting the previous

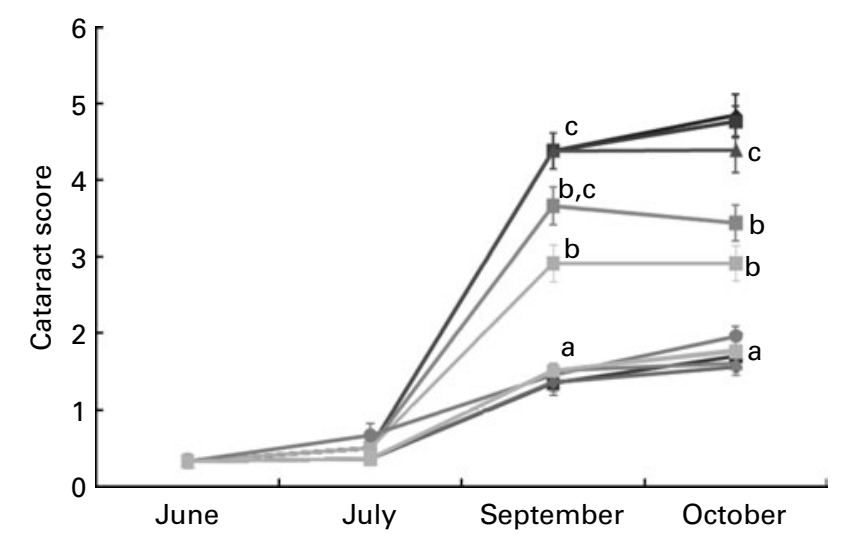

Fig. 2. Development of cataracts (mean cataract score of both eyes; $0-8$ ) in Atlantic salmon fed the experimental histidine diets: low (L), medium (M) and high $(H)$ (see Fig. 1) during three periods from June to October (mean of thirty examined fish per experimental duplicate). ${ }^{a, b, c}$ Mean values with unlike letters were significantly different $(P<0.05$; nested ANOVA and Tukey's honestly significant difference post hoc test). $\longrightarrow$, LLL; $\rightarrow$, LLM; $\longrightarrow$, LLH; $\longrightarrow$, LMM; - , LHH;,-- MMM; $-\longrightarrow, \mathrm{HLL} ; \multimap$, dietary histidine feeding regimes. Fish groups fed a diet that was not supplemented with histidine (L) from June to July developed significantly more cataract (mean score of 4.4 (SEM 0.2)) than fish fed M- or H-histidine diets, both with scores $<1.5 \quad(P<0.05)$. The cataract scores were also influenced by the feeding levels of histidine from July to September, where fish crossed over from $\mathrm{L}$ to $\mathrm{H}$ histidine diet (LH) showed significantly lower cataract score (2.9 (SEM 0.2)) than the L histidine group (LL), while fish crossed over from $\mathrm{L}$ to $\mathrm{M}$ histidine diet (LM) showed an intermediate higher score (3.7 (SEM 0.2)). No further development in cataract was observed from September to October in any group, ending with approximately similar cataract severities as observed in September. All the groups fed diets supplemented with histidine during the first feeding periods $(\mathrm{H}, \mathrm{M}$ and respective crossover diets) showed low but moderately increased cataract scores from the start $(0 \cdot 4)$ to the end ( $\leq 2 \cdot 0 ;$ Fig. 2$)$.

Considering all individually examined fish at the end of the experiment, no difference in cataract scores was observed between sexually mature fish and immature fish.

\section{Free amino acid composition in muscle}

Free histidine in white muscle tissue reflected systematically the feed histidine regimes, both in dietary concentration and feeding time (Fig. 3). In July, before the cataract outbreak, there were clear differences between the groups fed the $\mathrm{L}(0.13 \mu \mathrm{mol} / \mathrm{g})-$, $\mathrm{M}(0.70 \mu \mathrm{mol} / \mathrm{g})-$ and $\mathrm{H}(1.86 \mu \mathrm{mol} / \mathrm{g})$-histidine diets $(P<0.05)$, while in September after the outbreak, muscle histidine levels were closer in concentration with significantly higher concentrations in fish continuously fed H-histidine diet $(\mathrm{HH})$ than in fish continuously fed the L-histidine diet (LL). Fish crossed over to the $\mathrm{H}$ (LH)- and L-histidine diets (HL) showed intermediate muscle histidine levels. In October, after a second crossover, larger and systematic variations were observed among the eleven dietary groups; largely dividing into three concentration levels and reflecting the dietary histidine levels during the final period (Fig. 3).

White muscle anserine concentration constituted between 45 and $50 \%$ of the free amino acids and was less variable

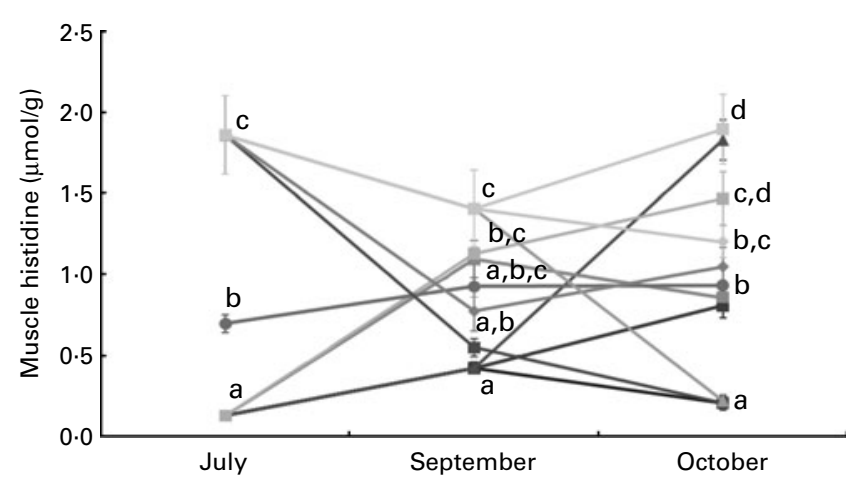

Fig. 3. Development of white muscle histidine in Atlantic salmon fed the experimental histidine diets: low $(\mathrm{L})$, medium $(\mathrm{M})$ and high $(\mathrm{H})$ during three periods from June to October. Data are expressed as means with their standard errors (six per experimental duplicate; see Fig. 1). ${ }^{a-d}$ Mean values with unlike letters were significantly different $(P<0.05$; nested ANOVA and Tukey's honestly significant difference post hoc test). $\longrightarrow$, LLL; $\longrightarrow$,

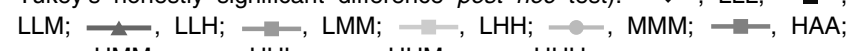


Table 2. Somatic data from duplicate groups of adult Atlantic salmon fed three levels of dietary His for 5 weeks before a massive outbreak of cataract (Mean values with their standard errors)

\begin{tabular}{|c|c|c|c|c|c|c|c|c|c|c|c|c|c|}
\hline \multirow[b]{3}{*}{ Feed code* } & \multicolumn{6}{|c|}{ Somatic data } & \multirow[b]{3}{*}{$n$} & \multicolumn{6}{|c|}{ Cataract score } \\
\hline & \multicolumn{2}{|c|}{ Weight $(\mathrm{g}) \dagger$} & \multicolumn{2}{|c|}{ Length $(\mathrm{cm})$} & \multicolumn{2}{|c|}{ CF (unit) } & & \multicolumn{2}{|c|}{ Left eye $(0-4)$} & \multicolumn{2}{|c|}{ Right eye (0-4) } & \multicolumn{2}{|c|}{ Total $(0-8)$} \\
\hline & Mean & SEM & Mean & SEM & Mean & SEM & & Mean & SEM & Mean & SEM & Mean & SEM \\
\hline L & 2251 & 62 & $57 \cdot 3$ & 0.5 & $1 \cdot 18$ & 0.01 & 58 & 0.3 & 0.1 & 0.2 & 0.1 & 0.5 & 0.1 \\
\hline M & 2135 & 61 & $56 \cdot 3$ & 0.5 & $1 \cdot 18$ & 0.02 & 56 & 0.4 & 0.1 & 0.3 & 0.1 & 0.7 & 0.2 \\
\hline $\mathrm{H}$ & 2200 & 59 & $57 \cdot 1$ & 0.4 & $1 \cdot 17$ & 0.02 & 58 & 0.2 & 0.1 & 0.2 & $0 \cdot 1$ & 0.4 & 0.1 \\
\hline ANOVA & \multicolumn{2}{|c|}{ NS } & \multicolumn{2}{|c|}{ NS } & \multicolumn{2}{|c|}{ NS } & & \multicolumn{2}{|c|}{ NS } & \multicolumn{2}{|c|}{ NS } & \multicolumn{2}{|c|}{ NS } \\
\hline
\end{tabular}

L, low; M, medium; $\mathrm{H}$, high.

* The single capital letter codes indicate the use of diets $\mathrm{L}, \mathrm{M}$ or $\mathrm{H}$ in the period from June to July.

$\dagger$ Initial values: weight, $1662 \mathrm{~g}$ ( $n$ 1834).

$\ddagger$ The data were evaluated by nested ANOVA and Tukey's honestly significant difference post hoc test (NS: $P>0.05$ ).

(6-8\% difference) among the dietary histidine groups (Table 4; Fig. 4). Muscle anserine was significantly higher in fish fed $\mathrm{M}(17.0 \mu \mathrm{mol} / \mathrm{g})$ - and $\mathrm{H}(16.7 \mu \mathrm{mol} / \mathrm{g})$-histidine diets, than in fish fed the unsupplemented histidine diet $(15.7 \mu \mathrm{mol} / \mathrm{g})$. Muscle anserine increased moderately from July to September in all the groups, while for fish crossed over from $\mathrm{L}$ to $\mathrm{H}$ histidine diets $(\mathrm{LH})$, this increase was significant. Moderate declines in muscle anserine were apparent from September to October, mostly in fish continuously fed the L-histidine diet (LLL; Fig. 4).

Muscle carnosine was significantly higher in the $\mathrm{M}$ than in the L histidine group at the first sampling in July (Table 4), and declined in all the groups in September (Table 5) except in fish crossed over from $\mathrm{L}$ to $\mathrm{M}$ histidine diet (LM), however, with no significant differences between the groups. Finally, carnosine increased during the last feeding period in all the groups except those fed the L-histidine diet during the final

Table 3. Somatic data from duplicate groups of adult Atlantic salmon fed three levels of dietary His levels for 5 months*

(Mean values with their standard errors)

\begin{tabular}{|c|c|c|c|c|c|c|c|}
\hline \multirow[b]{2}{*}{ Feed code $†$} & \multicolumn{2}{|c|}{ Weight (g) } & \multicolumn{2}{|c|}{ Length (cm) } & \multicolumn{2}{|c|}{$\begin{array}{l}\text { Condition } \\
\text { factor }\end{array}$} & \multirow[b]{2}{*}{$n$} \\
\hline & Mean & SEM & Mean & SEM & Mean & SEM & \\
\hline LLL & $2893^{a, b}$ & 129 & $61 \cdot 3$ & 0.7 & 1.22 & 0.03 & 47 \\
\hline LLM & $2850^{a, b}$ & 90 & $61 \cdot 8$ & 0.5 & 1.19 & 0.02 & 61 \\
\hline LLH & $2903^{a, b}$ & 82 & $62 \cdot 3$ & 0.4 & 1.19 & 0.02 & 60 \\
\hline LMM & $2842^{a, b}$ & 100 & $61 \cdot 6$ & 0.6 & 1.21 & 0.03 & 45 \\
\hline $\mathrm{LHH}$ & $2931^{a, b}$ & 109 & $62 \cdot 6$ & 0.5 & 1.18 & 0.03 & 48 \\
\hline MMM & $2785^{a}$ & 68 & $61 \cdot 3$ & 0.4 & 1.19 & 0.02 & 113 \\
\hline HLL & $3083^{a, b}$ & 114 & $62 \cdot 6$ & 0.5 & 1.24 & 0.03 & 47 \\
\hline HMM & $2682^{\mathrm{a}}$ & 90 & 60.7 & 0.5 & 1.18 & 0.02 & 52 \\
\hline $\mathrm{HHL}$ & $2935^{a, b}$ & 86 & 61.6 & 0.5 & 1.24 & 0.02 & 72 \\
\hline HHM & $2996^{a, b}$ & 85 & $62 \cdot 1$ & 0.4 & 1.23 & 0.02 & 64 \\
\hline $\mathrm{HHH}$ & $3207^{b}$ & 111 & $63 \cdot 1$ & 0.5 & $1 \cdot 26$ & 0.02 & 42 \\
\hline ANOVA $\ddagger$ & \multicolumn{2}{|c|}{$P<0.05$} & \multicolumn{2}{|c|}{ NS } & \multicolumn{2}{|c|}{ NS } & \\
\hline
\end{tabular}

L, low; M, medium; $\mathrm{H}$, high.

${ }^{a, b}$ Mean values within a column with unlike superscript letters were significantly different $(P<0.05)$.

* The experimental period was divided into three seasons (the three capital letter code indicates diets L, M and $\mathrm{H}$ used in June-July, July-September and September-October, respectively)

$\dagger$ Three-letter codes indicate the use of diets $\mathrm{L}, \mathrm{M}$ or $\mathrm{H}$ in the first, second and third periods.

¥ The data were evaluated by nested ANOVA and Tukey's honestly significant difference post hoc test (NS: $P>0.05$ ).
Table 4. Free amino acids in white muscle tissue $(\mu \mathrm{mol} / \mathrm{g})$ of adult Atlantic salmon fed low $(\mathrm{L} ; 9.3 \mathrm{~g} / \mathrm{kg})$, medium $(\mathrm{M} ; 12.8 \mathrm{~g} / \mathrm{kg})$ and high $(\mathrm{H} ; 17.2 \mathrm{~g} / \mathrm{kg}$ ) levels of histidine sampled in July (week 6) before a serious outbreak of cataract*

\begin{tabular}{|c|c|c|c|c|}
\hline \multirow[b]{2}{*}{ Amino acid } & \multicolumn{3}{|c|}{ Diets† } & \multirow{2}{*}{$\begin{array}{c}\text { Pooled } \\
\text { SEM }\end{array}$} \\
\hline & L & M & $\mathrm{H}$ & \\
\hline \multicolumn{5}{|l|}{ Essential } \\
\hline Thr & $0.43^{a}$ & $0.29^{b}$ & $0.29^{b}$ & 0.01 \\
\hline Val & 0.26 & 0.22 & 0.24 & 0.01 \\
\hline Met & 0.08 & 0.07 & 0.08 & 0.00 \\
\hline lle & $0.11^{a}$ & $0.08^{\mathrm{b}}$ & $0.09^{a, b}$ & 0.00 \\
\hline Leu & $0.21^{\mathrm{a}}$ & $0 \cdot 16^{\mathrm{b}}$ & $0.19^{a, b}$ & 0.01 \\
\hline Phe & $0 \cdot 12^{a, b}$ & $0 \cdot 10^{\mathrm{a}}$ & $0 \cdot 12^{\mathrm{b}}$ & 0.00 \\
\hline Lys & $0 \cdot 77^{\mathrm{a}}$ & $0.55^{\mathrm{b}}$ & $0.43^{\mathrm{b}}$ & 0.04 \\
\hline His & $0 \cdot 13^{a}$ & $0.70^{\mathrm{b}}$ & $1 \cdot 86^{\mathrm{C}}$ & 0.14 \\
\hline Arg & 0.15 & 0.12 & 0.12 & 0.01 \\
\hline Trp & ND & ND & ND & - \\
\hline \multicolumn{5}{|l|}{ Non-essential } \\
\hline Ser & $0.31^{a}$ & $0 \cdot 13^{b}$ & $0.20^{a, b}$ & 0.03 \\
\hline Glu & $1 \cdot 14^{\mathrm{a}}$ & $0.92^{a, b}$ & $0.90^{\mathrm{b}}$ & 0.04 \\
\hline Gln & 0.23 & 0.25 & 0.17 & 0.02 \\
\hline Pro & $0 \cdot 18$ & 0.15 & 0.15 & 0.01 \\
\hline Gly & $1.84^{\mathrm{a}}$ & $1.26^{\mathrm{b}}$ & $1.02^{\mathrm{b}}$ & 0.08 \\
\hline Ala & $2 \cdot 28^{\mathrm{a}}$ & $2 \cdot 16^{\mathrm{a}, \mathrm{b}}$ & $1.94^{\mathrm{b}}$ & 0.05 \\
\hline Tyr & $0.21^{a}$ & $0.14^{\mathrm{b}}$ & $0.22^{\mathrm{a}}$ & 0.01 \\
\hline Asp & $0 \cdot 13^{a, b}$ & $0 \cdot 15^{\mathrm{a}}$ & $0 \cdot 11^{\mathrm{b}}$ & 0.01 \\
\hline Hydroxyproline & $0.52^{\mathrm{a}}$ & $0.36^{b}$ & $0.22^{c}$ & 0.03 \\
\hline \multicolumn{5}{|l|}{ Nitrogenous compounds } \\
\hline$\beta$-Alanine & $0.38^{a}$ & $0.23^{b}$ & $0.23^{\mathrm{b}}$ & 0.02 \\
\hline 1-Methyl-histidine & 0.15 & 0.10 & 0.12 & 0.01 \\
\hline Carnosine & $0 \cdot 13^{\mathrm{a}}$ & $0.39^{\mathrm{b}}$ & $0.34^{a, b}$ & 0.04 \\
\hline Anserine & $15 \cdot 7^{\mathrm{a}}$ & $17 \cdot 0^{\mathrm{b}}$ & $16 \cdot 7^{\mathrm{b}}$ & 0.2 \\
\hline Taurine & 2.03 & 1.88 & 1.81 & $0 \cdot 18$ \\
\hline O-phospho-ethanolamine & 0.05 & 0.05 & 0.06 & $0.00 \ddagger$ \\
\hline Urea & 0.60 & 0.54 & 0.64 & 0.03 \\
\hline L- $\alpha$-Amino- $N$-butyric acid & $0.04^{a}$ & $0.02^{b}$ & $0.01^{b}$ & 0.00 \\
\hline Ethanolamine & 0.13 & 0.12 & 0.12 & 0.00 \\
\hline Ammonia & $4.93^{\mathrm{a}}$ & $4 \cdot 68^{\mathrm{a}, \mathrm{b}}$ & $4.49^{\mathrm{b}}$ & 0.05 \\
\hline Ornithine & $0.03^{a}$ & $0.02^{\mathrm{b}}$ & $0.02^{b}$ & 0.00 \\
\hline ¿Free amino acids & 34.5 & 34.0 & 34.2 & 0.3 \\
\hline
\end{tabular}

ND, below the detection limit.

${ }^{a, b, c}$ Mean values within a row with unlike superscript letters were significantly different $(P<0.05)$.

* Six samples per duplicate; evaluated by nested ANOVA and Tukey's honestly significant difference post hoc test at a level of $P<0.05$.

$\dagger$ Single-letter codes indicate the use of diets $\mathrm{L}, \mathrm{M}$ or $\mathrm{H}$ in the first feeding period. $\ddagger$ Pooled SEM $<0.005$. 


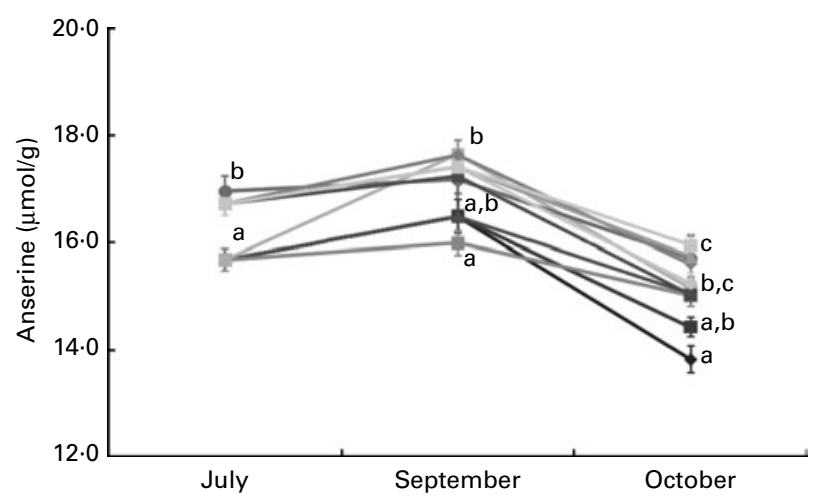

Fig. 4. Development of white muscle anserine in Atlantic salmon fed the experimental histidine diets: low $(\mathrm{L})$, medium $(\mathrm{M})$ and high $(\mathrm{H})$ during three periods from June to October. Data are expressed as means with their standard errors (six per experimental duplicate; see Fig. 1). ${ }^{a, b, c}$ Mean values with unlike letters were significantly different $(P<0.05$; nested ANOVA and Tukey's honestly significant difference post hoc test). $\longrightarrow$, LLL; $\longrightarrow$, $\mathrm{LLM} ; \longrightarrow$, LLH; $\longrightarrow, \mathrm{LMM} ;-1 \mathrm{LHH} ;-, \mathrm{MMM} ;-$, HLL; period $(<0 \cdot 2 \mu \mathrm{mol} / \mathrm{g})$. Muscle carnosine was four times higher in fish crossed over from $\mathrm{L}$ to $\mathrm{H}$ histidine diet after the second period (LLH; $P<0.05$ ).

After 6 weeks of feeding, several free amino acids in white muscle tissue, including some essential amino acids (threonine, isoleucine, leucine and lysine), were significantly higher in salmon fed the L-histidine diet compared with fish fed histidine-supplemented diets ( $M$ and $\mathrm{H}$; Table 4). The sum of free amino acids was, however, the same in all the dietary groups. After 13 weeks and following the cataract outbreak, there were minor differences among the other free amino acids in muscle than the imidazoles, with non-systematic differences in $O$-phospho-ethanolamine, aspartic acid, glutamine, alanine, tyrosine and ammonia $(P<0.05)$ among the groups (Table 5). Again, total amino acid concentration was equal in the samplings, between 34 and $35 \mu \mathrm{mol} / \mathrm{g}$. At the end of the trial, a profile similar to previous free amino acid profile in muscle was observed (data not shown), with significant higher concentration of selected amino acids (hydroxyproline, proline, threonine, glutamate, tyrosine, phenylalanine, ornithine, 1-methyl histidine) in salmon fed

Table 5. Free amino acids in white muscle tissue ( $\mu \mathrm{mol} / \mathrm{g})$ of adult Atlantic salmon fed low $(\mathrm{L} ; 9.3 \mathrm{~g} / \mathrm{kg}), \mathrm{medium}(\mathrm{M} ; 12.8 \mathrm{~g} / \mathrm{kg})$ and high $(\mathrm{H} ; 17.2 \mathrm{~g} / \mathrm{kg})$ levels of His in two periods and sampled in September after a serious outbreak of cataract*

\begin{tabular}{|c|c|c|c|c|c|c|c|c|}
\hline \multirow[b]{2}{*}{ Amino acid } & \multicolumn{7}{|c|}{ Diets $†$} & \multirow[b]{2}{*}{ Pooled SEM } \\
\hline & LL & LM & $\mathrm{LH}$ & $\mathrm{MM}$ & $\mathrm{HL}$ & $\mathrm{HM}$ & $\mathrm{HH}$ & \\
\hline \multicolumn{9}{|l|}{ Essential } \\
\hline Thr & 0.34 & 0.36 & 0.25 & 0.30 & 0.36 & 0.32 & 0.25 & 0.01 \\
\hline Val & 0.28 & 0.23 & 0.23 & 0.23 & 0.25 & 0.27 & 0.21 & 0.01 \\
\hline Met & 0.08 & 0.08 & 0.07 & 0.07 & 0.08 & 0.08 & 0.06 & 0.00 \\
\hline Ile & 0.12 & $0 \cdot 10$ & 0.10 & 0.10 & 0.12 & 0.12 & 0.08 & 0.00 \\
\hline Leu & 0.21 & 0.17 & 0.16 & 0.17 & 0.20 & 0.20 & 0.15 & 0.01 \\
\hline Phe & 0.08 & 0.08 & 0.06 & 0.07 & 0.08 & 0.08 & 0.06 & 0.00 \\
\hline Lys & 0.60 & 0.71 & 0.47 & 0.51 & 0.67 & 0.54 & 0.59 & 0.04 \\
\hline $\mathrm{His}$ & $0.42^{b}$ & $1.09^{a, b, c}$ & $1 \cdot 13^{\mathrm{a}, \mathrm{c}}$ & $0.93^{a, b, c}$ & $0.55^{\mathrm{b}, \mathrm{c}}$ & $0.77^{\mathrm{a}, \mathrm{b}, \mathrm{c}}$ & $1.40^{\mathrm{a}}$ & 0.07 \\
\hline Arg & 0.12 & 0.15 & 0.11 & 0.11 & 0.16 & 0.11 & 0.16 & 0.01 \\
\hline Trp & ND & ND & ND & ND & ND & ND & ND & - \\
\hline \multicolumn{9}{|l|}{ Non-essential } \\
\hline Ser & 0.38 & $0 \cdot 26$ & 0.33 & 0.29 & 0.39 & 0.35 & $0 \cdot 21$ & 0.03 \\
\hline Glu & $2 \cdot 14$ & $1 \cdot 61$ & $1 \cdot 70$ & 1.49 & $1 \cdot 78$ & 1.63 & 1.48 & 0.08 \\
\hline Gln & $0 \cdot 34^{a, b}$ & $0.36^{a, b}$ & $0.54^{\mathrm{b}, \mathrm{c}}$ & $0.47^{\mathrm{a}, \mathrm{b}, \mathrm{c}}$ & $0.60^{c}$ & $0.30^{\mathrm{a}}$ & $0.37^{a, b}$ & 0.03 \\
\hline Pro & 0.10 & 0.11 & 0.08 & 0.09 & 0.11 & $0 \cdot 10$ & 0.08 & 0.00 \\
\hline Gly & $2 \cdot 14$ & 1.79 & 1.93 & $1 \cdot 74$ & 2.02 & 1.79 & 1.63 & 0.05 \\
\hline Ala & $1.94^{\mathrm{a}, \mathrm{b}}$ & $2 \cdot 25^{\mathrm{a}}$ & $1.83^{b}$ & $1.90^{a, b}$ & $2 \cdot 02^{\mathrm{a}, \mathrm{b}}$ & $2 \cdot 13^{\mathrm{a}, \mathrm{b}}$ & $1.75^{\mathrm{b}}$ & 0.04 \\
\hline Tyr & $0.09^{a, b}$ & $0 \cdot 10^{a}$ & $0.05^{\mathrm{b}}$ & $0.06^{a, b}$ & $0.08^{a, b}$ & $0.09^{a, b}$ & $0.06^{a, b}$ & 0.00 \\
\hline Asp & $0 \cdot 14^{a, b}$ & $0 \cdot 16^{a, b}$ & $0 \cdot 19^{a}$ & $0.19^{a}$ & $0.19^{a}$ & $0 \cdot 15^{\mathrm{a}, \mathrm{b}}$ & $0.13^{b}$ & 0.01 \\
\hline Hydroxyproline & 0.19 & 0.29 & 0.19 & 0.24 & 0.26 & 0.22 & 0.21 & 0.01 \\
\hline \multicolumn{9}{|l|}{ Nitrogenous compounds } \\
\hline$\beta$-Alanine & 0.21 & $0 \cdot 21$ & $0 \cdot 16$ & 0.16 & 0.18 & 0.17 & $0 \cdot 16$ & 0.01 \\
\hline 1-Methyl-histidine & $0.13^{\mathrm{e}}$ & $0.12^{\mathrm{a}, \mathrm{c}}$ & $0 \cdot 10^{\mathrm{a}, \mathrm{b}, \mathrm{c}}$ & $0.09^{b}$ & $0 \cdot 11^{a, b, c}$ & $0.11^{a, c}$ & $0 \cdot 12^{\mathrm{a}, \mathrm{d}, \mathrm{e}}$ & 0.00 \\
\hline Carnosine & 0.07 & 0.22 & 0.11 & 0.11 & 0.08 & 0.06 & 0.09 & 0.02 \\
\hline Anserine & $16 \cdot 5^{\mathrm{a}, \mathrm{b}, \mathrm{c}}$ & $16 \cdot 0^{a}$ & $17 \cdot 6^{\mathrm{b}, \mathrm{c}}$ & $17 \cdot 2^{a, b, c}$ & $17 \cdot 2^{\mathrm{b}, \mathrm{c}}$ & $17 \cdot 6^{\mathrm{c}}$ & $17 \cdot 4^{\mathrm{b}, \mathrm{c}, \mathrm{d}}$ & 0.1 \\
\hline Taurine & $1 \cdot 20$ & $1 \cdot 23$ & $1 \cdot 16$ & $1 \cdot 18$ & 1.03 & $1 \cdot 30$ & $1 \cdot 20$ & 0.04 \\
\hline O-phospho-ethanolamine & $0.05^{a, b}$ & 0.05 & $0.04^{b}$ & $0.05^{a, b}$ & $0.05^{a, b}$ & $0.05^{a}$ & $0.05^{a, b}$ & $0.00 \ddagger$ \\
\hline Urea & 0.77 & 0.60 & 0.69 & 0.69 & 0.66 & 0.62 & 0.56 & 0.04 \\
\hline L- $\alpha$-Amino- $N$-butyric acid & 0.03 & 0.03 & 0.03 & 0.03 & 0.03 & 0.03 & 0.02 & 0.00 \\
\hline Ethanolamine & 0.20 & 0.18 & 0.19 & 0.17 & 0.17 & 0.21 & 0.21 & 0.01 \\
\hline Ammonia & $4.94^{a, d}$ & $5 \cdot 14^{a}$ & $4 \cdot 36^{c}$ & $4 \cdot 50^{\mathrm{b}, \mathrm{c}}$ & $4 \cdot 46^{\mathrm{b}, \mathrm{c}}$ & $4 \cdot 80^{\mathrm{a}, \mathrm{b}}$ & $4 \cdot 66^{\mathrm{b}, \mathrm{c}, \mathrm{d}}$ & 0.05 \\
\hline Ornithine & 0.02 & 0.03 & 0.01 & 0.02 & 0.02 & 0.02 & 0.02 & 0.00 \\
\hline$\Sigma$ Free amino acids & $35 \cdot 0$ & $35 \cdot 0$ & $35 \cdot 2$ & $34 \cdot 4$ & $35 \cdot 2$ & 35.5 & $34 \cdot 6$ & 0.2 \\
\hline
\end{tabular}

ND, below the detection limit.

${ }^{a-e}$ Mean values within a row with unlike superscript letters were significantly different $(P<0.05)$

* Six samples per duplicate; evaluated by nested ANOVA and Tukey's honestly significant difference post hoc test at a level of $P<0.05$.

$\dagger$ Two-letter codes indicate the use of diets $\mathrm{L}, \mathrm{M}$ or $\mathrm{H}$ in the first and second feeding periods.

$\ddagger$ Pooled SEM $<0.005$. 


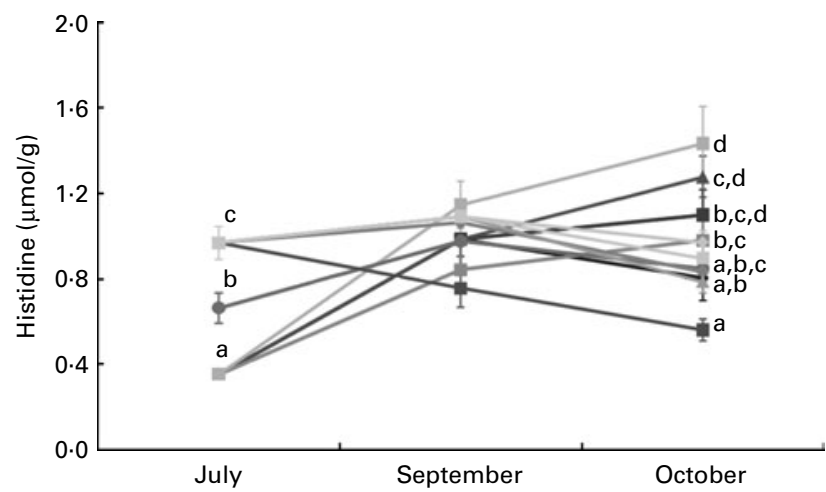

Fig. 5. Development of lens histidine in Atlantic salmon fed the experimental histidine diets: low $(\mathrm{L})$, medium $(\mathrm{M})$ and high $(\mathrm{H})$ during three periods from June to October. Data are expressed as means with their standard errors (six per experimental duplicate; see Fig. 1). ${ }^{a, b, c, d}$ Mean values with unlike letters were significantly different $(P<0.05$; nested ANOVA and Tukey's honestly significant difference post hoc test). $\longrightarrow$, LLL; $\rightarrow-$, LLM;

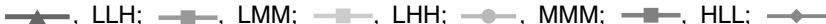
$\mathrm{HMM} ; \longrightarrow, \mathrm{HHL} \longrightarrow, \mathrm{HHM} ; \longrightarrow, \mathrm{HHH}$.

the L-histidine diet during the two last feeding periods (HLL and LLL groups) compared with the other groups. Total amino acid concentration was equal within the sampling, but somewhat lower than that observed previously $(31-33 \mu \mathrm{mol} / \mathrm{g})$.

\section{Lens amino acid composition}

As in the white muscle, lens histidine and NAH reflected dietary histidine concentrations strictly systematically relative to both concentration and feeding period (Figs. 5 and 6). Large differences in the concentration of NAH between the feeding groups was observed in July before the cataract outbreak, with significant differences $(P<0 \cdot 05)$ between all the three groups (Fig. 6). All the dietary groups had increased lens NAH from July to September, except fish crossed over from a $\mathrm{H}$ to $\mathrm{L}$ histidine diet (HL). The lens NAH increases were most significant in fish with a low NAH status in July, and depended on dietary histidine concentration in this second feeding period.

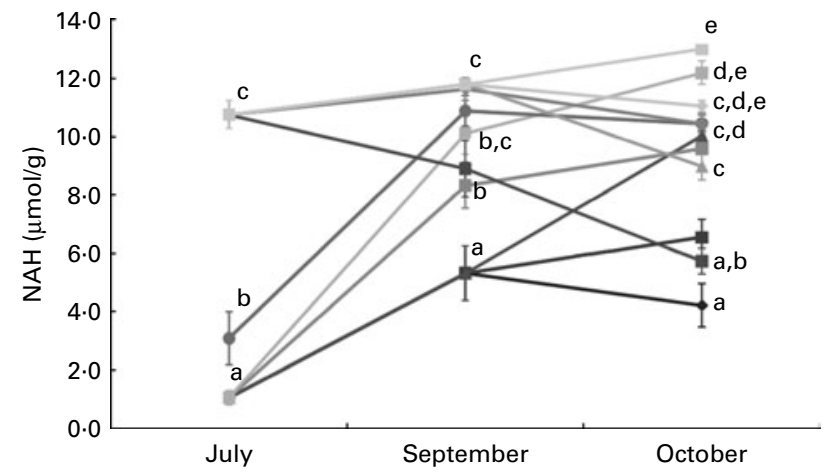

Fig. 6. Development of lens $\mathrm{N}$-acetyl-histidine (NAH) in Atlantic salmon fed the experimental histidine diets: low $(L)$, medium $(M)$ and high $(H)$ during three periods from June to October. Data are represented as means with their standard errors (six per experimental duplicate; see Fig. 1). $\mathrm{a}, \mathrm{b}, \mathrm{c}, \mathrm{d}, \mathrm{e}$ Mean values with unlike letters were significantly different $(P<0.05$; nested ANOVA and Tukey's honestly significant difference post hoc test). $\longrightarrow$, LLL; $\longrightarrow$, LLM; $\longrightarrow, \mathrm{LLH} ;-\square, \mathrm{LMM} ;-\square, \mathrm{LHH} ;-$

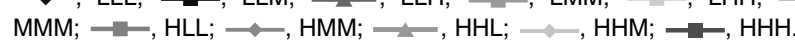

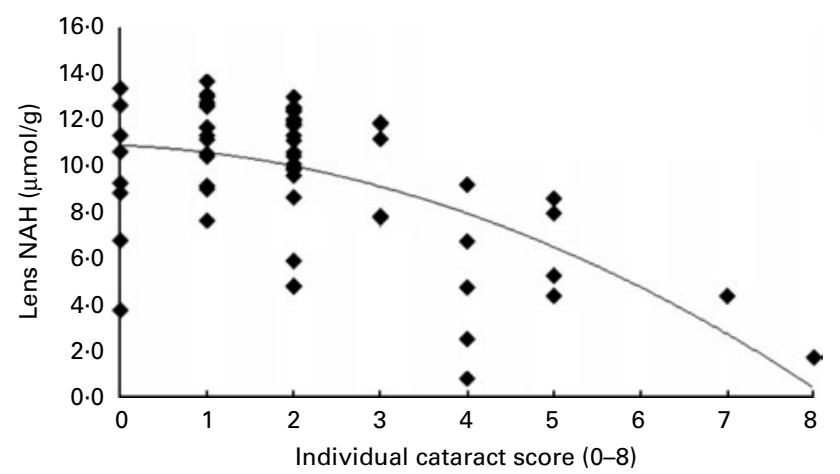

Fig. 7. Correlation between individual lens $\mathrm{N}$-acetyl-histidine (NAH) and cataract scores in adult Atlantic salmon fed experimental diets differing in histidine from June to September and examined after the second period with a serious outbreak of cataract $(n 60) . y=-0.1439 x^{2}-0.1624 x+10.913$; $R^{2} 0.3556$.

In August, the lens NAH status correlated negatively with severity of cataract given as cataract score (Fig. 7). At the end of the trial, lens NAH showed a 3-fold difference between the groups, depending on dietary histidine feeding regimes (Fig. 6). The H-histidine diet obviously supported saturation level of lens $\mathrm{NAH}$ at $11-12 \mu \mathrm{mol} / \mathrm{g}$.

Concentrations of most free amino acids in the lens were significantly higher in the group fed L histidine compared with the group fed $\mathrm{M}$ and $\mathrm{H}$ histidine before the cataract outbreak (Table 6). In same manner as the sum of free amino acids, concentration of selected free amino acids like hydroxyproline, glutamate, alanine, isoleucine, leucine, tyrosine, phenylalanine and 1-methyl histidine gradually decreased in fish fed L-, M- and H-histidine diets. Adding NAH to the other lens amino acids gave, however, a total amino acid level of $24.3 \mu \mathrm{mol} / \mathrm{g}$ in $\mathrm{L}$ and $\mathrm{H}$ histidine groups, and somewhat lower level in lenses from the $M$ histidine group $(20 \cdot 1 \mu \mathrm{mol} / \mathrm{g})$. In September, after the outbreak of cataract, a similar elevated concentration of major amino acids were observed in the L histidine group (LL) compared with the groups fed histidine-supplemented diets in either of the two periods (Table 7). Again, the total amino acid, including $\mathrm{NAH}$, in lens was similar among the groups within the range of $23.3-25.4 \mu \mathrm{mol} / \mathrm{g}$. At the conclusion of the trial in October, free amino acids like glutamate, glycine, phenylalanine, taurine and 1-methyl histidine in lens were still elevated in salmon fed continuously on the L-histidine diet (LLL), especially compared with fish fed the H-histidine $\operatorname{diet}(\mathrm{HHH})(P<0 \cdot 05$; data not shown).

\section{Discussion}

The present study demonstrates for the first time that adult Atlantic salmon have a practical requirement for dietary histidine levels above present recommendations by Norwegian Research Council ${ }^{(10)}$ to prevent cataract development during their second year in seawater. Low prevalence and severity of cataracts were recorded at the start of the experiment. A massive outbreak of cataracts was recorded in September, probably initiated by a period of increased water temperature in July. Moderately elevated and fluctuating temperatures have been demonstrated to represent risk factors for cataract development, both experimentally and in field ${ }^{(27,28)}$, probably 
Table 6. Free amino acids in lens tissue of adult Atlantic salmon fed low (L; $9.3 \mathrm{~g} / \mathrm{kg})$, medium (M; $12.8 \mathrm{~g} / \mathrm{kg}$ ) and high (M; $17.2 \mathrm{~g} / \mathrm{kg}$ ) levels of His sampled in July (week 6) before a serious outbreak of cataract*

\begin{tabular}{|c|c|c|c|c|}
\hline \multirow[b]{2}{*}{ Amino acid } & \multicolumn{3}{|c|}{ Diets† } & \multirow{2}{*}{$\begin{array}{l}\text { Poolec } \\
\text { SEM }\end{array}$} \\
\hline & L & M & $\mathrm{H}$ & \\
\hline \multicolumn{5}{|l|}{ Essential } \\
\hline Thr & $0.38^{a}$ & $0.24^{\mathrm{b}}$ & $0.24^{b}$ & 0.01 \\
\hline Val & $1.61^{\mathrm{a}}$ & $1.35^{\mathrm{a}}$ & $0.91^{\mathrm{b}}$ & 0.07 \\
\hline Met & $2 \cdot 27^{\mathrm{a}}$ & $2 \cdot 26^{\mathrm{a}}$ & $1 \cdot 74^{\mathrm{b}}$ & 0.09 \\
\hline lle & $0.70^{\mathrm{a}}$ & $0.46^{\mathrm{b}}$ & $0.27^{c}$ & 0.04 \\
\hline Leu & $2 \cdot 03^{\mathrm{a}}$ & $1.56^{\mathrm{b}}$ & $0.93^{\mathrm{c}}$ & 0.09 \\
\hline Phe & $2 \cdot 33^{\mathrm{a}}$ & $1.84^{\mathrm{b}}$ & $1.29^{\mathrm{C}}$ & 0.10 \\
\hline Lys & $0.22^{\mathrm{a}}$ & $0.14^{\mathrm{b}}$ & $0 \cdot 13^{\mathrm{b}}$ & 0.01 \\
\hline His & $0 \cdot 16^{a}$ & $0.50^{\mathrm{b}}$ & $0.80^{c}$ & 0.05 \\
\hline $\operatorname{Arg}$ & ND & ND & ND & - \\
\hline Trp & $0.38^{a}$ & $0.19^{\mathrm{b}}$ & $0 \cdot 13^{b}$ & 0.02 \\
\hline \multicolumn{5}{|l|}{ Non-essential } \\
\hline Ser & $0.42^{\mathrm{a}}$ & $0.25^{\mathrm{b}}$ & $0.25^{\mathrm{b}}$ & 0.02 \\
\hline Glu & $1.68^{a}$ & $1.45^{\mathrm{b}}$ & $1 \cdot 25^{\mathrm{c}}$ & 0.04 \\
\hline Gln & $1.37^{\mathrm{a}}$ & $0.32^{\mathrm{b}}$ & $0 \cdot 14^{\mathrm{b}}$ & 0.11 \\
\hline Pro & $0 \cdot 11^{a}$ & $0.08^{\mathrm{b}}$ & $0.07^{b}$ & $0.00 \ddagger$ \\
\hline Gly & $1.07^{\mathrm{a}}$ & $0.60^{\mathrm{b}}$ & $0.60^{\mathrm{b}}$ & 0.05 \\
\hline Ala & $0.71^{\mathrm{a}}$ & $0.55^{\mathrm{b}}$ & $0.43^{\mathrm{C}}$ & 0.03 \\
\hline Tyr & $1 \cdot 12^{\mathrm{a}}$ & $0.74^{\mathrm{b}}$ & $0.46^{\mathrm{C}}$ & 0.06 \\
\hline Asp & $0.30^{\mathrm{a}}$ & $0.20^{\mathrm{b}}$ & $0 \cdot 16^{\mathrm{b}}$ & 0.01 \\
\hline Hydroxyproline & $0.20^{\mathrm{a}}$ & $0 \cdot 17^{a, b}$ & $0 \cdot 14^{\mathrm{b}}$ & 0.01 \\
\hline Cys & 0.09 & 0.04 & 0.05 & 0.01 \\
\hline Asn & $0 \cdot 10^{\mathrm{a}}$ & $0.06^{\mathrm{b}}$ & $0.04^{\mathrm{b}}$ & 0.01 \\
\hline \multicolumn{5}{|l|}{ Nitrogenous compounds } \\
\hline$\beta$-Alanine & 0.24 & 0.25 & 0.22 & 0.01 \\
\hline 1-Methyl-histidine & $0.08^{a}$ & $0.06^{a, b}$ & $0.04^{b}$ & 0.00 \\
\hline Taurine & $1 \cdot 11^{\mathrm{a}}$ & $1 \cdot 15^{\mathrm{a}}$ & $0.80^{\mathrm{b}}$ & 0.04 \\
\hline O-phospho-ethanolamine & $0.70^{\mathrm{a}}$ & $0.65^{\mathrm{a}}$ & $0.48^{\mathrm{b}}$ & 0.02 \\
\hline Urea & $0.79^{a}$ & $0.50^{\mathrm{b}}$ & $0.59^{a, b}$ & 0.05 \\
\hline L- $\alpha$-Amino- $N$-butyric acid & $0.11^{\mathrm{a}}$ & $0.08^{\mathrm{b}}$ & $0.07^{b}$ & 0.00 \\
\hline Ammonia & $0 \cdot 15^{a}$ & $0.03^{b}$ & $0.02^{b}$ & 0.01 \\
\hline Phospho-serine & $0 \cdot 10^{\mathrm{a}}$ & $0.08^{\mathrm{a}}$ & $0.06^{b}$ & 0.00 \\
\hline Sarcosine & $1.63^{a}$ & $0.38^{\mathrm{b}}$ & $0.38^{b}$ & 0.15 \\
\hline$\alpha$-Amino adipic acid & $0 \cdot 18^{a}$ & $0.17^{\mathrm{a}}$ & $0 \cdot 12^{\mathrm{b}}$ & 0.01 \\
\hline Cystathionine 1 & 0.03 & 0.03 & 0.02 & 0.01 \\
\hline Cystathionine 2 & $0.37^{a}$ & $0.14^{\mathrm{b}}$ & $0 \cdot 14^{\mathrm{b}}$ & 0.02 \\
\hline ¿Free amino acids & $23 \cdot 2^{\mathrm{a}}$ & $17 \cdot 0^{\mathrm{b}}$ & $13.5^{\mathrm{c}}$ & 0.76 \\
\hline $\mathrm{NAH}$ & $1.05^{\mathrm{a}}$ & $3.09^{b}$ & $10 \cdot 8^{\mathrm{c}}$ & 0.79 \\
\hline $\begin{array}{l}\Sigma \text { Free amino } \\
\text { acids including } \mathrm{NAH}\end{array}$ & $24 \cdot 3$ & $20 \cdot 1$ & $24 \cdot 3$ & \\
\hline
\end{tabular}

$\mathrm{ND}$, below the detection limit; $\mathrm{NAH}, \mathrm{N}$-acetyl-histidine.

${ }_{a, b, c}$ Mean values within a row with unlike superscript letters were significantly different $(P<0.05)$.

* Six samples per duplicate; evaluated by nested ANOVA and Tukey's honestly significant difference post hoc test at a level of $P<0.05$

† Single-letter codes indicate the use of diets $\mathrm{L}, \mathrm{M}$ or $\mathrm{H}$ in the first feeding period. $\ddagger$ Pooled SEM $<0.005$.

related to increased growth and metabolism. The cataract severity among the dietary groups was systematically related to the dietary histidine level and histidine feeding period. All the groups of salmon fed the unsupplemented diet with $9 \mathrm{~g}$ histidine/kg during the first period developed severe cataract, while histidine-supplemented diets $\geq 13 \mathrm{~g}$ histidine $/ \mathrm{kg}$, as in the M- and H-histidine diets prevented cataract. However, fish crossed over from low to both histidine-supplemented diets (LM and LH groups) developed intermediate severities of cataract, suggesting that feeding a histidine-supplemented diet from onset July was too late for a maximum cataract preventive effect. On the other hand, fish crossed over from the H-histidine diet in the first period to a L-histidine diet in the actual period with cataract outbreak (HL group) were fully protected. In summary, both dietary histidine supplementation and histidine feeding period were critical for cataract prevention. Since the present histidine supplementation levels ( $\geq 13 \mathrm{~g}$ histidine $/ \mathrm{kg}$ ) prevented cataract in adult Atlantic salmon after a relatively short period of feeding, this represents an effective counter measure in periods with increased risk for cataract, like rises in water temperature during the summer season.

In a previous study with Atlantic salmon smolts, dietary histidine levels of 12 and $18 \mathrm{~g} / \mathrm{kg}$ were compared, and showed that the former was not able to prevent cataract during a 26-week feeding period ${ }^{(7)}$. This was a first indication that histidine requirement to support growth and health in Atlantic salmon parr and smolts under practical conditions may be higher than the given minimum requirement of $7 \mathrm{~g} /$ $\mathrm{kg}^{(10)}$. Salmonids undergo smoltification to prepare for a migration from fresh water to seawater, and muscle and eye histidine metabolism seems to be a part of these adaptations $^{(7,20)}$. Feeding studies with Atlantic salmon throughout the parr-smolt transformation indicated that the histidine requirements may vary according to the physiological status of the fish. After transferring to seawater and when provided with sufficient dietary His support, Atlantic salmon seems to build up tissue-specific histidine compounds, anserine and NAH in muscle and lens, respectively, thereby 'trapping' histidine intracellularly and making it unavailable for protein synthesis ${ }^{(7)}$. These specific histidine compounds are believed to have important physiological and biochemical stabilising functions, related to cataract development. The underlying historical studies for establishing the histidine requirement in salmonids $^{(10)}$ did not consider these tissue imidazoles as markers for histidine status.

With feed target composition to fulfil the requirements for optimal growth and protein utilisation, amino acid deficiencies have not been considered to be an area of concern with respect to fish health ${ }^{(22)}$. Somatic growth seemed not to vary according to the present dietary histidine regimes. All the diets had levels well above the minimum requirement for histidine, and all the dietary groups showed reasonable growth during the experiment (specific growth rate $\geq 040 \% / d$ ). Low temperature fishmeal and soyabean protein concentrates represent high protein qualities with highly digestible histidine ${ }^{(10)}$. Likewise, the free supplemented histidine salt is highly digestible and shows superior absorption ${ }^{(6,7,12)}$. However, the present study shows that the use of a fishmeal-based diet without histidine supplementation did not supply sufficient histidine to fulfil essential functions of histidine beyond protein synthesis.

A significant part of the fish population $(28 \%)$ in all net pens had initiated sexual maturation during the trial. Excluding these individuals at the final sampling generally improved growth rates, but did not affect the overall outcome of growth among the experimental groups. Breck et al. ${ }^{(7)}$ suggested a growth-promoting effect of histidine in salmon smolts, which was argued to be related to direct effects on amino acid metabolism or by improved physiological conditions of the tissue. A similar growth promotion could not be confirmed in adult salmon in the present study. The growth and macronutrient deposition differ, however, between smolts and growers, which may mask the potential modest effects of histidine on protein synthesis ${ }^{(12)}$. 
Table 7. Free amino acids in lens tissue of adult Atlantic salmon fed low (L; $9.3 \mathrm{~g} / \mathrm{kg}$ ), medium (M; $12.8 \mathrm{~g} / \mathrm{kg})$ and high (M; $17.2 \mathrm{~g} / \mathrm{kg})$ levels of His sampled in September (week 13) after the outbreak of cataract*

\begin{tabular}{|c|c|c|c|c|c|c|c|c|}
\hline \multirow[b]{2}{*}{ Amino acid } & \multicolumn{7}{|c|}{ Diets $\dagger$} & \multirow[b]{2}{*}{ Pooled SEM } \\
\hline & LL & LM & $\mathrm{LH}$ & MM & $\mathrm{HL}$ & $\mathrm{HM}$ & $\mathrm{HH}$ & \\
\hline \multicolumn{9}{|l|}{ Essential } \\
\hline Thr & $0.35^{a}$ & $0.24^{a, b}$ & $0 \cdot 24^{a, b}$ & $0.22^{a, b}$ & $0.24^{a, b}$ & $0.23^{a, b}$ & $0 \cdot 18^{b}$ & 0.01 \\
\hline Val & 1.41 & 0.97 & $1 \cdot 14$ & 0.94 & 1.21 & 1.14 & 1.01 & 0.05 \\
\hline Met & 1.03 & $1 \cdot 31$ & 1.02 & $1 \cdot 20$ & 1.21 & 0.95 & 0.93 & 0.04 \\
\hline lle & $0.65^{a}$ & $0.42^{a, b}$ & $0.48^{a, b}$ & $0.36^{b}$ & $0.48^{a, b}$ & $0.46^{a, b}$ & $0.38^{b}$ & 0.02 \\
\hline Leu & 1.44 & $1 \cdot 10$ & $1 \cdot 10$ & 0.99 & 1.30 & $1 \cdot 13$ & 0.98 & 0.05 \\
\hline Phe & 0.87 & 0.94 & 0.62 & 0.68 & 0.90 & 0.55 & 0.50 & 0.05 \\
\hline Lys & 0.20 & 0.14 & $0 \cdot 17$ & 0.13 & 0.15 & $0 \cdot 17$ & 0.15 & 0.01 \\
\hline His & 0.73 & 0.67 & 0.95 & 0.80 & 0.62 & 0.94 & 0.92 & 0.03 \\
\hline Arg & $0.05^{\mathrm{a}}$ & $0.02^{\mathrm{b}}$ & $0.02^{b}$ & $0.01^{\mathrm{b}}$ & $0.01^{\mathrm{b}}$ & $0.02^{\mathrm{b}}$ & $0.01^{\mathrm{b}}$ & $0.00 \ddagger$ \\
\hline Trp & $0.39^{a}$ & $0.24^{b}$ & $0.18^{\mathrm{b}}$ & $0.13^{b}$ & $0.21^{b}$ & $0.12^{b}$ & $0.12^{\mathrm{b}}$ & 0.02 \\
\hline \multicolumn{9}{|l|}{ Non-essential } \\
\hline Ser & $0.44^{\mathrm{a}}$ & $0.23^{b}$ & $0.27^{\mathrm{b}}$ & $0.20^{\mathrm{b}}$ & $0.23^{b}$ & $0.22^{b}$ & $0.19^{b}$ & 0.01 \\
\hline Glu & 1.56 & 1.72 & 1.61 & 1.53 & 1.55 & 1.63 & 1.53 & 0.03 \\
\hline Gln & 0.95 & 0.66 & 0.61 & 0.50 & 0.49 & 0.57 & 0.62 & 0.04 \\
\hline Pro & $0.16^{a}$ & $0.07^{\mathrm{b}}$ & $0.08^{\mathrm{b}}$ & $0.05^{\mathrm{b}}$ & $0.06^{b}$ & $0.06^{b}$ & $0.05^{\mathrm{b}}$ & 0.01 \\
\hline Gly & $1 \cdot 20^{\mathrm{a}}$ & $0.68^{\mathrm{b}}$ & $0.63^{b}$ & $0.68^{\mathrm{b}}$ & $0.72^{\mathrm{b}}$ & $0.67^{\mathrm{b}}$ & $0.51^{\mathrm{b}}$ & 0.05 \\
\hline Ala & $0.58^{a}$ & $0.47^{a, b}$ & $0.45^{a, b}$ & $0.37^{b}$ & $0.35^{b}$ & $0.39^{a, b}$ & $0.37^{b}$ & 0.02 \\
\hline Tyr & 0.84 & 0.82 & 0.66 & 0.68 & 0.74 & 0.66 & 0.65 & 0.02 \\
\hline Asp & $0 \cdot 18$ & 0.16 & 0.15 & 0.13 & 0.14 & $0 \cdot 12$ & 0.13 & 0.01 \\
\hline Hydroxyproline & $0 \cdot 13^{a, b}$ & $0 \cdot 12^{a, b}$ & $0 \cdot 10^{\mathrm{b}}$ & $0 \cdot 12^{a, b}$ & $0.15^{\mathrm{a}}$ & $0 \cdot 10^{\mathrm{b}}$ & $0 \cdot 10^{\mathrm{b}}$ & 0.00 \\
\hline Cys & $0.12^{\mathrm{a}}$ & $0.04^{\mathrm{a}, \mathrm{b}}$ & $0.06^{a, b}$ & $0.03^{a, b}$ & $0.02^{\mathrm{b}}$ & $0.03^{a, b}$ & $0.03^{a, b}$ & 0.01 \\
\hline Asp & $0.10^{\mathrm{a}}$ & $0.05^{a, b, c}$ & $0 \cdot 10^{a, c}$ & $0.04^{\mathrm{b}}$ & $0.05^{a, b, c}$ & $0.08^{a, b, c}$ & $0.09^{a, b, c}$ & 0.01 \\
\hline \multicolumn{9}{|l|}{ Nitrogenous compounds } \\
\hline B-Alanine & $0.16^{\mathrm{b}}$ & $0.20^{a}$ & $0.13^{\mathrm{b}}$ & $0.16^{a, b}$ & $0.15^{\mathrm{b}}$ & $0.15^{\mathrm{b}}$ & $0.15^{\mathrm{b}}$ & 0.00 \\
\hline 1-Methyl-histidine & $0.07^{\mathrm{a}}$ & $0.05^{a, b}$ & $0.03^{b}$ & $0.03^{b}$ & $0.03^{\mathrm{b}}$ & $0.02^{\mathrm{b}}$ & $0.03^{b}$ & 0.00 \\
\hline Taurine & $1.33^{\mathrm{a}}$ & $1.05^{\mathrm{a}, \mathrm{b}}$ & $0.82^{b, c}$ & $0.62^{c}$ & $0.82^{b, c}$ & $0.66^{b, c}$ & $0.69^{b, c}$ & 0.04 \\
\hline O-phospho-ethanolamine & 0.75 & 0.83 & 0.66 & 0.82 & 0.83 & 0.78 & 0.75 & 0.02 \\
\hline Urea & $0.61^{a}$ & $0.30^{\mathrm{b}}$ & $0.45^{\mathrm{a}, \mathrm{b}}$ & $0.44^{\mathrm{a}, \mathrm{b}}$ & $0.34^{\mathrm{b}}$ & $0.42^{\mathrm{a}, \mathrm{b}}$ & $0.40^{\mathrm{a}, \mathrm{b}}$ & 0.02 \\
\hline L- $\alpha$-Amino- $N$-butyric acid & $0 \cdot 15^{a}$ & $0 \cdot 11^{\mathrm{b}}$ & $0 \cdot 12^{a, b}$ & $0.09^{\mathrm{b}}$ & $0 \cdot 12^{\mathrm{a}, \mathrm{b}}$ & $0 \cdot 11^{\mathrm{b}}$ & $0.09^{b}$ & 0.00 \\
\hline Ammonia & $0.08^{\mathrm{b}}$ & $0.08^{\mathrm{b}}$ & $0.06^{a, b}$ & $0.02^{\mathrm{a}}$ & $0.08^{b}$ & $0.05^{a, b}$ & $0.06^{a, b}$ & 0.01 \\
\hline Phospho-serine & $0.08^{a}$ & $0.05^{a, b}$ & $0.04^{\mathrm{b}}$ & $0.04^{b}$ & $0.06^{a, b}$ & $0.04^{\mathrm{b}}$ & $0.04^{\mathrm{b}}$ & 0.00 \\
\hline Sarcosine & $0.71^{a}$ & $0.42^{a, b}$ & $0.44^{a, b}$ & $0.55^{a, b}$ & $0.41^{a, b}$ & $0.50^{a, b}$ & $0.39^{b}$ & 0.03 \\
\hline$\alpha$-Amino adipic acid & 0.13 & 0.14 & 0.14 & 0.15 & 0.17 & 0.19 & 0.16 & 0.01 \\
\hline Cystathionine 1 & 0.07 & 0.04 & 0.08 & 0.07 & 0.05 & 0.08 & 0.07 & 0.01 \\
\hline Cystathionine 2 & 0.11 & $0 \cdot 11$ & $0 \cdot 12$ & 0.09 & 0.10 & 0.09 & 0.11 & 0.00 \\
\hline 3-Methyl-histidine & 0.01 & 0.02 & 0.01 & 0.02 & 0.02 & 0.01 & 0.01 & 0.00 \\
\hline ¿Free amino acids & $18 \cdot 1^{a}$ & $15 \cdot 0^{a, b}$ & $14 \cdot 2^{\mathrm{b}}$ & $13 \cdot 4^{b}$ & $14 \cdot 5^{\mathrm{b}}$ & $13 \cdot 8^{\mathrm{b}}$ & $12 \cdot 9^{\mathrm{b}}$ & 0.33 \\
\hline $\mathrm{NAH}$ & $5 \cdot 3^{\mathrm{a}}$ & $8 \cdot 3^{b}$ & $10 \cdot 1^{\mathrm{b}, \mathrm{c}}$ & $10 \cdot 9^{b, c}$ & $8 \cdot 9^{\mathrm{b}, \mathrm{c}}$ & $11 \cdot 6^{\mathrm{c}}$ & $11 \cdot 8^{\mathrm{c}}$ & 0.35 \\
\hline ¿Free amino acids including $\mathrm{NAH}$ & 23.4 & $23 \cdot 3$ & $24 \cdot 3$ & $24 \cdot 3$ & 23.4 & $25 \cdot 4$ & $24 \cdot 7$ & \\
\hline
\end{tabular}

$\mathrm{NAH}, \mathrm{N}$-acetyl-histidine.

${ }_{\mathrm{a}, \mathrm{b}, \mathrm{c}}$ Mean values within a row with unlike superscript letters were significantly different $(P<0.05)$.

${ }^{*} n 6$ samples per duplicate; evaluated by nested ANOVA and Tukey's honestly significant difference post hoc test at a level of $P<0.05$.

$\dagger$ Two-letter codes indicate the use of diet L, $\mathrm{M}$ or $\mathrm{H}$ in the first and second feeding periods.

$\ddagger$ Pooled SEM $<0.005$.

White muscle anserine, representing the major imidazole in salmon body, was affected significantly by dietary histidine regimes but within a narrow concentration range. According to the present high muscle concentrations and minor changes between groups and samplings, anserine synthesis seems to be prioritised in all the groups, even in groups with critical histidine supply and status during the outbreak of cataract. Between September and October, muscle anserine generally declined moderately, with a concomitant rise in precursors to anserine biosynthesis (carnosine and $\beta$-alanine) in most groups, except those fed on a L-histidine diet. This indicates a compensatory up-regulation of anserine synthesis in the final period, however, again depending on available free histidine as substrate. While elevated muscle anserine during parr-smolt transformation reflects a need for buffering capacity relative to increased anaerobic burst swimming activity in seawater, anserine has also been suggested to be a more important antioxidant in rainbow trout growers than juveniles ${ }^{(15)}$. Maximal muscle tissue concentration of anserine was achieved by use of the M-histidine diet with $12.8 \mathrm{~g}$ histidine $/ \mathrm{kg}$. Muscle carnosine, representing a low concentration imidazole intermediate in anserine biosynthesis was generally higher in concentration before than after the outbreak of cataract. In July, there seemed to be a saturation in anserine synthesis in both fish fed $\mathrm{M}$ and $\mathrm{H}$ histidine-supplemented diets, as evaluated by significantly lower $\beta$-alanine and carnosine and higher anserine concentrations in these two groups compared with fish fed the L-histidine diet. At later samplings, both anserine precursors were lower in all the groups.

Low concentration levels of NAH in the lens have been considered a direct risk factor for cataract development ${ }^{(7)}$. Dietary levels of $17.6 \mathrm{~g}$ histidine $/ \mathrm{kg}$ mitigated cataract 
formation in salmon smolts in their first season in seawater compared with a control diet with $11.7 \mathrm{~g}$ histidine $/ \mathrm{kg}$, representing lens NAH concentrations of $>10$ and $<2 \mu \mathrm{mol} / \mathrm{g}$, respectively. In the present study, the lens $\mathrm{NAH}$ reflected the L-, M- and H-histidine diets by showing mean values of $1 \cdot 1,3 \cdot 1$ and $10 \cdot 8 \mu \mathrm{mol} / \mathrm{g}$ before the outbreak of cataract. During the outbreak of cataract, the concentrations increased to $5.3,10.9$ and $11.8 \mu \mathrm{mol} / \mathrm{g}$, respectively, illustrating an active build-up of lens NAH in all the groups; however, the concentrations were not increased sufficiently in the L histidine groups. This confirms the applicability of NAH as an indicator for cataract susceptibility, in adult salmon. The intermediate histidine feed level (M), increasing lens NAH from $3 \cdot 1$ to $10 \cdot 9 \mu \mathrm{mol} / \mathrm{g}$ from July to September was sufficient to prevent cataract during the outbreak. Similarly, fish crossed over from $\mathrm{H}$ to $\mathrm{L}$ histidine diet (HL), with lens NAH between 9 and $11 \mu \mathrm{mol} / \mathrm{g}$ in September showed equal cataract prevention. In contrast, fish crossed over from a low to a histidinesupplemented diet (LM or LH) showed more severe cataract development; despite lens $\mathrm{NAH}$ levels $>8 \mu \mathrm{mol} / \mathrm{g}$ at the time of sampling. Clearly, the actual status of $\mathrm{NAH}$ in these groups during the first and second feeding periods may have changed between the samplings, and for the latter groups, this represented a critical low NAH concentration which consequently led to cataract development. Since the status of lens NAH was not recorded exactly at the outbreak of cataract, it is not possible to estimate a critical value of this indicator to prevent cataract. Mean cataract scores did not increase much from September to October, suggesting that the major outbreak was over in September. For individual fish sampled in September, a negative correlation was apparent between lens NAH concentration and cataract scores (Fig. 7), as was observed previously in salmon smolts after transferring to seawater ${ }^{(7)}$. Generally, a low level of NAH in the lens seems to represent a lower defence towards fluctuations in both external and biotic factors, which eventually $\mathrm{Pb}$ to cataract changes. The relatively wide concentration ranges at each score level indicates that the individual fish may be at different phases of cataract development and repair at the time of sampling.

The mechanisms behind the cataract alleviating effect of histidine are not fully understood. Histidine and its related imidazoles have been suggested to cover many biochemical roles in the salmon tissues besides being an essential amino acid in protein synthesis, such as lens osmoregulation ${ }^{(7)}$. The former has been given support by recent studies on salmon lens ex vivo cultures ${ }^{(29)}$, where exposure of lenses to a hypoosmotic medium caused an efflux of $\mathrm{NAH}$ from the lens and into the medium. This finding was interpreted as a survival mechanism by the lens; to adjust osmolality according to the surrounding medium to prevent swelling and eventually rupture of the lens capsule. In the present study, free amino acid profiles in muscle and lens were recorded both before and after the cataract outbreak to determine if any other amino acid was influenced by the histidine feeding regimes. These data indicate first that the muscle and lens did not suffer from deficiency of any other essential amino acids (methionine, cystine, tryptophan) that have been related to cataract development ${ }^{(1)}$. Secondly, the concentrations of many free amino acids were significantly elevated in the $\mathrm{L}$ histidine groups compared with the histidine-supplemented groups, most probably to equalise osmolality or the total concentration of amino acids in deficiency of histidine for NAH synthesis. This latter argument is also supporting the role of NAH in lens osmoregulation. Obviously, NAH synthesis represents trapping of high concentrations of histidine for use as an effective osmolyte, compared with a less efficient mixed battery of other amino acids, among others taurine, glutamic acid, glutamine, glycine, valine, methionine, leucine, tyrosine and phenylalanine; all with concentrations $>1 \mu \mathrm{mol} / \mathrm{g}$ in the $\mathrm{L}$ histidine group before the cataract outbreak and somewhat less after the outbreak. This is summarised in the Tables 6 and 7 as a fairly equal sum of total amino acids (including NAH) in the lens and supports our earlier finding in salmon smolts ${ }^{(7)}$.

In summary, the present study demonstrates for the first time a histidine-related cataract in adult Atlantic salmon in their second year in seawater. Low lens NAH concentrations in the first two feeding periods coincided with increased severity of cataract formation. Feeding a diet with $12.8 \mathrm{~g}$ histidine/ $\mathrm{kg}$ throughout the experimental periods prevented cataract development. Alternatively, it also seemed possible to prevent cataract by pre-feeding a H-histidine diet in risk periods. Even lately initiated cataract counter measures seem to reduce cataract outbreaks, where a diet supporting $17.2 \mathrm{~g}$ histidine $/ \mathrm{kg}$ added more protection compared with a diet with $12.8 \mathrm{~g}$ histidine $/ \mathrm{kg}$. A risk factor in the present study was probably a rapid rise in the sea temperature during summer. The outcome of the present histidine feeding study may be used as a nutritional guideline for cataract prevention in adult salmonids in periods of increased risks.

In a separate communication, we aimed to explore potential molecular mechanisms for the present histidinerelated cataract, both with respect to acute cataractogenesis from July to September and a suggested repair phase from September to October. Cataract $v$. non-cataract lenses from selected groups were screened for differentially expressed genes using a microarray experiment ${ }^{(25)}$. The expression levels of a subset of genes coding for proteins involved in antioxidation, osmoregulation, carbohydrate metabolism and apoptosis were identified, all representing the mechanisms involved in cataractogenesis and potentially influenced by histidine status.

\section{Acknowledgements}

The authors wish to thank research engineer Johnny Elvrum at Skretting ARC, Lerang Research station, and engineer Anita Birkenes at National Institute of Nutrition and Seafood Research, for their valuable technical assistance at the rearing site and the laboratory, respectively. This research was supported by the Norwegian Research Council (\#168435/S40), Marine Harvest Norway, Skretting ARC, Norway, Skretting Ireland and National Institute of Nutrition and Seafood Research. There are no conflicts of interest that the authors should disclose. Each co-author has assisted the first author in preparing the manuscript, although they participated differently in designing the research (O. B., R. W., W. K. and R. F.), making the feeds (W. K. and R. F.), conducting the experiment and sampling (C. T., O. B., R. W., W. K. and R. F.), and performing chemical (C. T.) and statistical analyses (C. T., O. B. and R. W.). 


\section{References}

1. Bjerkås $\mathrm{E}$, Breck $\mathrm{O}$ \& Waagbø $\mathrm{R}$ (2006) The role of nutrition in cataract formation in farmed fish. CAB Rev Perspect Agric Vet Sci Nutr Nat Res 1, 1-16.

2. Hargis WG (1991) Disorders of the eye in finfish. Аnnu Rev Fish Dis 1, 95-117.

3. Ersdal C, Midtlyng PJ \& Jarp J (2001) An epidemiological study of cataracts in seawater farmed Atlantic salmon Salmo salar. Dis Aquat Organ 45, 229-236.

4. Menzies FD, Crockford T, Breck O, et al. (2002) Estimation of direct costs associated with cataracts in farmed Atlantic salmon (Salmo salar). Bull Eur Ass Fish Pathol 22, 27-32.

5. Bjerkås E \& Sveier $\mathrm{H}$ (2004) The influence of nutritional and environmental factors on osmoregulation and cataracts in Atlantic salmon (Salmo salar L.). Aquaculture 235, 101-122.

6. Breck $\mathrm{O}$, Bjerkås E, Campbell $\mathrm{P}$, et al. (2003) Cataract preventative role of mammalian blood meal, histidine, iron and zinc in diets for Atlantic salmon (Salmo salar L.) of different strains. Aquacult Nutr 9, 341-350.

7. Breck $\mathrm{O}$, Bjerkås E, Campbell $\mathrm{P}$, et al. (2005) Histidine nutrition and genotype affect cataract development in Atlantic salmon (Salmo salar L.). J Fish Dis 28, 357-371.

8. Wall AE (1998) Cataracts in farmed Atlantic salmon (Salmo salar) in Ireland, Norway and Scotland from 1995 to 1997. Vet Rec 142, 626-631.

9. EC (1990) Council Directive 90/667/EEC of 27 November 1990 laying down the veterinary rules for the disposal and processing of animal waste, for its placing on the market and for the prevention of pathogens in feedstuffs of animal or fish origin and amending Directive 90/425/EEC. In Official Journal L 363, 27/12/1990, pp. 0051-0060. Brussels: EC.

10. NRC (1993) Nutrient Requirements of Fish. Washington, DC: National Academy Press.

11. Baslow MH (1998) Function of the $N$-acetyl-L-histidine system in the vertebrate eye - evidence in support of a role as a molecular water pump. J Mol Neurosci 10, 193-208.

12. Breck $\mathrm{O}$, Bjerkås E, Sanderson J, et al. (2005) Dietary histidine affects lens protein turnover and synthesis of $\mathrm{N}$-acetylhistidine in Atlantic salmon (Salmo salar L.) undergoing parr-smolt transformation. Aquacult Nutr 11, 321-332.

13. Ogata HY (2002) Muscle buffering capacity of yellowtail fed diets supplemented with crystalline histidine. J Fish Biol 61, 1504-1512.

14. Suzuki T, Hirano $T$ \& Suyama M (1987) Free imidazole compounds in white and dark muscles of migratory marine fish. Comp Biochem Physiol 87B, 615-619.
15. Kim H, Park SY \& Choi YH (1999) Determination of the antioxidant anserine in muscle of juvenile and mature rainbow trout. Oncorhynchus mykiss. Arch Ryb Fish 7, 65-72.

16. Wade MA \& Tucker HN (1998) Antioxidant characteristics of L-histidine. J Nutr Biochem 9, 308-315.

17. Aldini G, Carini M, Beretta G, et al. (2002) Carnosine is a quencher of 4-hydroxy-nonenal: through what mechanism of reaction? Biochem Biophys Res Commun 298, 699-706.

18. Hipkiss AR (2000) Carnosine and protein carbonyl groups: a possible relationship. Biochemistry (Mosc) 65, 771-778.

19. Ogata HY, Konno S \& Silverstein JT (1998) Muscular buffering capacity of the parr and smolts in Oncorhynchus masou. Aquaculture 168, 303-310.

20. Munakata A, Aida K, Amano M, et al. (2000) Changes in histidine and anserine levels in hatchery-reared honmasu salmon parr after release in a river. J World Aquacult Soc 31, 274-278.

21. Baslow MH (1999) The existence of molecular water pumps in the nervous system: a review of the evidence. Neurochem Int 34, 77-90.

22. Hardy RW (2001) Nutritional deficiencies in commercial aquaculture: likelihood, onset, and identification. In Nutrition and Fish Health, pp. 131-147 [C Lim and CD Webster, editors]. Birminghamton, NY: Food Products Press.

23. EC (1998) Commission Directive 98/64/EC of 3 September 1998. Establishing community methods of analysis for the determination of aminoacids, crude oils and fats, and olaquindox in feedingstuffs and amending Directive 71/393/EEC. In Commission directive 98/64/EC. Brussels: EC.

24. Wall T \& Bjerkås E (1999) A simplified method of scoring cataracts in fish. Bull Eur Assoc Fish Pathol 19, 162-165.

25. Tröße C, Waagb $\varnothing$ R, Breck O, et al. (2009) Genome-wide transcription analysis of histidine-related cataract in Atlantic salmon (Salmo salar L.). Mol Vis 15, 1332-1350.

26. O'Dowd JJ, Cairns MT, Trainor M, et al. (1990) Analysis of carnosine, homocarnosine and other histidyl derivatives in rat brain. J Neurochem 55, 446-452.

27. Bjerkås E \& Bjørnestad E (1999) Is there a connection between rapid fluctuation in water temperature and cataract development in the Atlantic Salmon (Salmo salar L.)? Bull Eur Assoc Fish Pathol 19, 166-169.

28. Bjerkås E, Bjørnestad E, Breck O, et al. (2001) Water temperature regimes affect cataract development in smolting Atlantic salmon Salmo salar L. J Fish Dis 24, 281-291.

29. Tröße C, Rhodes JD, Sanderson J, et al. (2010) Effect of plant-based feed ingredients on osmoregulation in the Atlantic salmon lens. Comp Biochem Physiol Part B 155, 354-362. 